\title{
Binary Power Optimality for Two Link Full-Duplex Network
}

\author{
Shalanika Dayarathna, Rajitha Senanayake and Jamie Evans \\ Department of Electrical and Electronic Engineering, University of Melbourne, Australia \\ Email: sdayarathna@student.unimelb.edu.au,rajitha.senanayake@unimelb.edu.au,jse@unimelb.edu.au
}

\begin{abstract}
In this paper, we analyse the optimality of binary power allocation in a network that includes full-duplex communication links. Considering a network with four communicating nodes, two of them operating in half-duplex mode and the other two in full-duplex mode, we prove that binary power allocation is optimum for the full-duplex nodes when maximizing the sum rate. We also prove that, for half-duplex nodes binary power allocation is not optimum in general. However, for the two special cases, 1) the low signal-to-noise-plus-interference (SINR) regime and, 2) the approximation by the arithmetic mean-geometric mean inequality, binary power allocation is optimum for the approximated sum rate even for the half-duplex nodes. We further analyse a third special case using a symmetric network for which the optimum power allocation is binary, under a sufficient condition. Numerical examples are included to illustrate the accuracy of the results.
\end{abstract}

\section{INTRODUCTION}

Throughput optimization in wireless networks has been very important for decades. In a wireless network, if interference is treated as Gaussian noise, we can write the Shannon theoretic rate of a node as $\log (1+S I N R)$, with $S I N R$ representing the received signal-to-interference-plus-noise ratio of the given node [1]. Maximizing the sum rate, when links have maximum power constraints, is a difficult problem because of its nonlinear and non-convex nature [2].

One approach to solve this problem is the use of approximation techniques to develop sub-optimal algorithms through convexification and linearization [3], [4]. However, these approximations could introduce extra constraints that cause the resulting power vector to steer away from the optimum solution in certain cases. For example, in [5], [6] a high SINR approximation is used to establish convexity in the sum rate objective function, while in [4], Taylor expansion and arithmetic mean-geometric mean approximation in low SINR region is used to establish convexity in the sum rate objective function. The approximation by construction does not allow completely turning off the power of any link at any time, which causes significant sub-optimality in the resulting power vector in certain cases. Another common approach is the use of simple power allocation structures such as the binary power allocation. "Binary" here means that a link is either "on" or "off", with the transmitting node either operating at zero power, or maximum power, without taking any value in the continuum of possible values between zero and the maximum transmit power.

This work was supported by the Australian Research Council Discovery Project under Grant DP180101205 and Discovery Early Career Researcher Award under Grant DE180100501.
Interestingly, binary power allocation - as simple as it may sound - shows close to optimal results in certain interfering networks [4], [7]-[9]. More specifically, in [2], the authors consider the uplink of a single-cell where multiple users transmit to a single base station, and show, via the theory of majorization, that optimal power control is binary. In [9], the authors consider a symmetric interfering network where all direct links have one particular gain, and all the crosslinks have another particular gain and show that binary power allocation is optimal when such total symmetry is maintained.

The work mentioned above considers half-duplex networks where nodes cannot transmit and receive simultaneously using the same time/frequency resources. With the introduction of full-duplex mode, nodes are now allowed to transmit and receive simultaneously using the same time/frequency resources. In this paper, we focus on the optimality of binary power allocation in an interference network with full-duplex links when the objective is to maximize the sum rate.

Binary power allocation in full-duplex networks has received very little attention in the past [10]-[12]. In [10], [11] the authors consider a single-cell network with a full-duplex base station and two half-duplex users. By decomposing the network into two half-duplex links they show that binary power allocation is optimal when the objective is to maximize the sum rate. In [12], the authors consider a simple network with just two nodes operating in full-duplex mode and obtain a similar conclusion. They further generalize it to a multiuser network even for which the optimality of binary power allocation holds.

In the present paper we take a step further, and consider a network with four nodes where two operate in half-duplex mode while the other two operate in full-duplex mode. Such a network model can practically arise in device-to-device (D2D) communication system underlaying cellular communication [13]. Within this setting, we show that binary power allocation is optimal for the full-duplex nodes but it is not optimal for the half-duplex nodes in general. We analyse the binary power optimality of the half-duplex nodes under three special cases. Firstly, the low SINR regime where the approximation $\log _{2}(1+S I N R) \approx \frac{S I N R}{\ln _{2}}$ holds. Secondly, using the approximation by the arithmetic mean-geometric mean inequality and thirdly using a symmetric network for which binary power allocation is optimum under a sufficient condition. We include numerical results to further illustrate our results. 


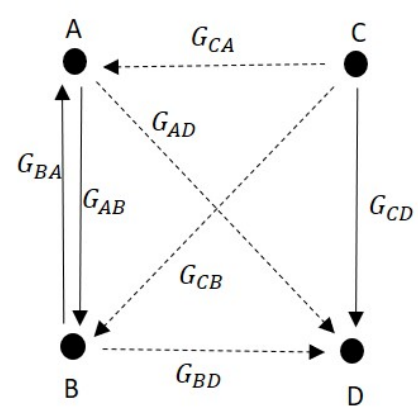

Figure 1: Extended Full-duplex network model

\section{System ModeL}

We consider a network with four communicating nodes as illustrated in Fig. 1, where the links between nodes $A$ and $B$ and nodes $C$ and $D$ are desired links while the rest are interference. Nodes $A$ and $B$ operate in full-duplex mode and can transmit and receive at the same time. Nodes $C$ and $D$ operate in half-duplex mode with node $C$ transmitting to node $D$. The link gains between nodes $i$ and $j$ are denoted by $G_{i j}$ where $i j \in\{A B, B A, C D, C A, A D, C B, B D\}$.

Based on the above notations, the received SINR of nodes $A, B$ and $D$ can be defined as

$$
\begin{aligned}
\gamma_{A} & =\frac{P_{B} G_{B A}}{\sigma^{2}+I_{A}+P_{C} G_{C A}}, \\
\gamma_{B} & =\frac{P_{A} G_{A B}}{\sigma^{2}+I_{B}+P_{C} G_{C B}}, \\
\gamma_{D} & =\frac{P_{C} G_{C D}}{\sigma^{2}+P_{A} G_{A D}+P_{B} G_{B D}},
\end{aligned}
$$

where $P_{A}, P_{B}$ and $P_{C}$ denote the transmit powers of nodes $A, B$ and $C$, respectively, $I_{A}$ and $I_{B}$ denote the residual selfinterference in nodes $A$ and $B$ and $\sigma^{2}$ is the noise power at nodes $A, B$ and $D$. Thus, the sum rate of the entire network can be defined as

$$
R=\log _{2}\left(1+\gamma_{A}\right)+\log _{2}\left(1+\gamma_{B}\right)+\log _{2}\left(1+\gamma_{D}\right) .
$$

\section{Binary Power Optimality}

We formulate an optimization problem to maximize the sum rate of the entire network as

$$
\begin{aligned}
& \max _{P_{A}, P_{B}, P_{C}} \log _{2}\left(\left(1+\gamma_{A}\right)\left(1+\gamma_{B}\right)\left(1+\gamma_{D}\right)\right) \\
& \text { Subject to } 0 \leq P_{A}, P_{B}, P_{C} \leq P_{\max },
\end{aligned}
$$

where $P_{\max }$ is the maximum power allowed per node. By considering the fact that $\log _{2}$ is a monotonically increasing function, the optimization in (2) can be further simplified as

$$
\begin{aligned}
& \max _{P_{A}, P_{B}, P_{C}}\left(1+\gamma_{A}\right)\left(1+\gamma_{B}\right)\left(1+\gamma_{D}\right) \\
& \text { Subject to } 0 \leq P_{A}, P_{B}, P_{C} \leq P_{\max } .
\end{aligned}
$$

Assuming that self-interference is treated as zero mean additive Gaussian noise, we model the variance of the residual self-interference when active as [14]

$$
I=\beta P_{i}^{\lambda},
$$

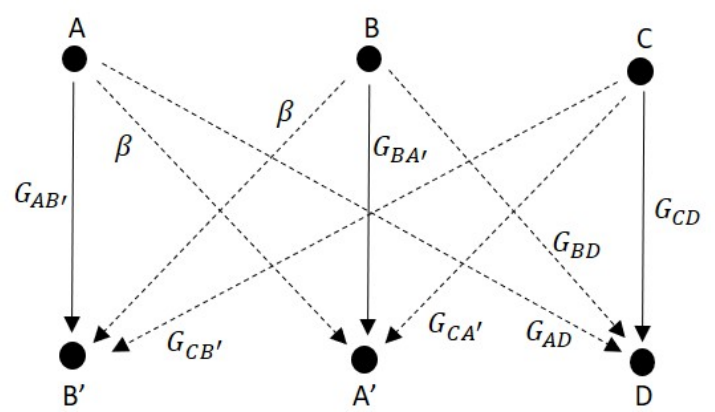

Figure 2: Half-duplex network with three node pairs where interference between two pairs are equal to each other.

where $P_{i}$ is the average power transmitted by the node $i$ when active and $\beta$ and $\lambda(0 \leq \lambda \leq 1)$ are constants that reflect the quality of the selected interference cancellation technique. Based on this general model, which is extremely hard to analyse, we extract two interesting models for the selfinterference when $\lambda=1$ and $\lambda=0$.

\section{A. Model I}

Under this model we set $\lambda=1$ and the residual selfinterference is modelled as zero mean additive Gaussian noise where variance increases linearly with the transmission power [15], [16]. Therefore, we can re-write the self-interference in nodes $A$ and $B$ as

$$
I_{x}=\beta P_{x}, \quad \forall P_{x} \geq 0, x \in\{A, B\} .
$$

Based on (5), the the sum rate maximization problem in (3) can be reformulated as

$$
\begin{aligned}
\max _{P_{A}, P_{B}, P_{C}}(1+ & \left.\frac{P_{B} G_{B A}}{\sigma^{2}+\beta P_{A}+P_{C} G_{C A}}\right) \\
& \times\left(1+\frac{P_{A} G_{A B}}{\sigma^{2}+\beta P_{B}+P_{C} G_{C B}}\right) \\
& \times\left(1+\frac{P_{C} G_{C D}}{\sigma^{2}+P_{A} G_{A D}+P_{B} G_{B D}}\right)
\end{aligned}
$$

Subject to $0 \leq P_{A}, P_{B}, P_{C} \leq P_{\max }$.

The optimization in (6) becomes equivalent to the sum rate optimization of three half-duplex node pairs where interference between two pairs are equal to each other, as illustrated in Fig. 2. In [4], the authors have shown that for a general half-duplex network with node pairs, $N>2$ binary power allocation is not optimum but it yields only a negligible capacity loss when compared to the optimal solution resulting from continuous power control. We can conclude that these results hold for the special case where two lowest interference gains, i.e., the self interference $\beta$, are equal as indicated by the example below.

Example 1: Consider a half-duplex network with three node pairs similar to Fig. 2 and following parameters. Maximum and minimum power constraint of $P_{\max }=1$ and $P_{\min }=0$ as commonly used in literature [4]. Assuming identical noise figures for the different receivers, the additive white Gaussian noise (AWGN) power is found as $k T_{0} B$, where $k$ is the 
Boltzmann's constant, $T_{0}=290$ Kelvin is the ambient temperature, and $B=1 \mathrm{MHz}$ is the equivalent noise bandwidth, i.e, $\sigma^{2}=4.0039 \times 10^{-15}$. As an example of the randomly generated channel gains, let

$$
\left[\begin{array}{lll}
G_{A A^{\prime}} & G_{A B^{\prime}} & G_{A D} \\
G_{B A^{\prime}} & G_{B B^{\prime}} & G_{B D} \\
G_{C A^{\prime}} & G_{C B^{\prime}} & G_{C D}
\end{array}\right]=10^{-12} \times\left[\begin{array}{ccc}
\beta & 0.8 & 0.029 \\
0.7 & \beta & 0.091 \\
0.031 & 0.018 & 0.9
\end{array}\right],
$$

where residual self interference constant $\beta=0.009$. Then by the best binary power allocation $P_{A}=P_{B}=P_{C}=P_{\max }$, we can obtain a sum rate of $11.8697 \mathrm{bits} / \mathrm{Hz}$ while optimal power allocation of $P_{A}=P_{\max }, P_{B}=0.95 P_{\max }$ and $P_{C}=$ $0.29 P_{\max }$ gives a maximum achievable sum rate of 12.1603 bits/Hz.

Therefore, under this linear self-interference model, we can conclude that binary power allocation is not optimum for the full-duplex nodes $A$ and $B$ or the half-duplex node $C$ in general. However, we note that binary power allocation is optimum for the approximated sum rate in the low SINR regime, under the approximation $\log _{2}(1+S I N R) \approx \frac{S I N R}{\ln 2}$, as shown in [4].

\section{B. Model II}

When sufficient self-interference cancellation has been performed on the full-duplex nodes, we can assume that the residual self-interference is modelled as zero mean additive Gaussian noise, with the noise variance being independent of the transmission power of the full-duplex nodes [17]-[19] and we set $\lambda=0$. Therefore, we can re-write the noise plus self interference in nodes $A$ and $B$ as

$$
\sigma_{x}^{2}=\left\{\begin{array}{ll}
\sigma^{2}+\beta & P_{x}>0, \\
\sigma^{2} & P_{x}=0,
\end{array} \quad x \in\{A, B\} .\right.
$$

Then the sum rate maximization problem in (3) can be reformulated as

$$
\begin{aligned}
& \max _{P_{A}, P_{B}, P_{C}} \hat{R} \\
& \text { Subject to } 0 \leq P_{A}, P_{B}, P_{C} \leq P_{\max },
\end{aligned}
$$

where

$$
\begin{aligned}
& \hat{R}=\left(1+\frac{P_{B} G_{B A}}{\sigma_{A}^{2}+P_{C} G_{C A}}\right)\left(1+\frac{P_{A} G_{A B}}{\sigma_{B}^{2}+P_{C} G_{C B}}\right) \\
&\left(1+\frac{P_{C} G_{C D}}{\sigma^{2}+P_{A} G_{A D}+P_{B} G_{B D}}\right) .
\end{aligned}
$$

Next, we proceed to analyse the optimality of binary power allocation in different communication nodes with this selfinterference model.

\section{Binary Power Optimality of Full-Duplex Nodes}

Let us focus on the binary power optimality with respect to the full-duplex node $A$. Since the self-interference model in (7) is discontinuous let us first consider a continuous model for $P_{A}$ where $\sigma_{A}^{2}=\sigma^{2}+\beta, \forall P_{A} \geq 0$. The resulting $\hat{R}$ of this continuous self-interference model is denoted as $\hat{R}_{\text {con }}$. Taking the partial derivative of $\hat{R}_{c o n}$ with respect to $P_{A}$ we obtain,

$$
\frac{\partial \hat{R}_{c o n}}{\partial P_{A}}=\left(\sigma_{A}^{2}+P_{C} G_{C A}+P_{B} G_{B A}\right) \frac{a P_{A}^{2}+2 b P_{A}+c}{d},
$$

where,

$$
\begin{aligned}
a= & G_{A B} G_{A D}^{2}, \\
b= & G_{A B} G_{A D}\left(\sigma^{2}+P_{B} G_{B D}\right), \\
c= & G_{A B}\left(\sigma^{2}+P_{B} G_{B D}\right)^{2}-P_{C} G_{C D} G_{A D}\left(\sigma_{B}^{2}+P_{C} G_{C B}\right) \\
+ & P_{C} G_{A B} G_{C D}\left(\sigma^{2}+P_{B} G_{B D}\right), \\
d= & \left(\sigma^{2}+P_{A} G_{A D}+P_{B} G_{B D}\right)^{2}\left(\sigma_{A}^{2}+P_{C} G_{C A}\right) \\
& \left(\sigma_{B}^{2}+P_{C} G_{C B}\right) .
\end{aligned}
$$

Even though the sign of $c$ can not be determined, from the above expressions we can clearly see that the values of $a, b$ and $d$ are all positive. Note that, for the first derivative in (9) to be zero, the term $a P_{A}^{2}+2 b P_{A}+c$ has to be zero. Thus, the second derivative of $\hat{R}_{c o n}$ at $\partial \hat{R}_{c o n} / \partial P_{A}=0$ can be evaluated as

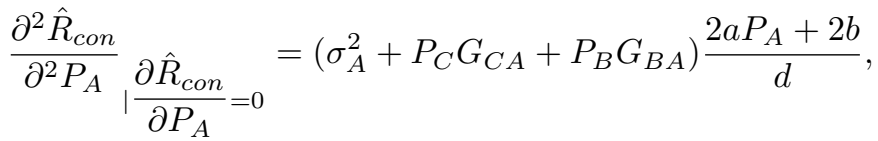

which is strictly positive. Having $\partial^{2} \hat{R}_{c o n} / \partial^{2} P_{A}>0$ at $\partial \hat{R}_{\text {con }} / \partial P_{A}=0$ indicates that the range $0 \leq P_{A} \leq P_{\max }$ can only has a global minimizer. Thus, within this range $\hat{R}_{\text {con }}$ is either increasing or decreasing or convex with respect to $P_{A}$. Therefore, maximum sum rate would be in the corner points $\left(0, P_{\max }\right)$ proving that optimum power allocation is binary.

To draw a conclusion about the binary optimality of $\hat{R}$ given in (8) we compare $\hat{R}$ with $\hat{R}_{\text {con }}$ and note that they are the same except at corner point $P_{A}=0$ where $\hat{R}>\hat{R}_{\text {con }}$. Since $\hat{R}_{\text {con }}$ is convex with respect to $P_{A}$, we can conclude that $\hat{R}$ remains convex as well. Thus, binary power allocation is optimum for $P_{A}$. Since the network is defined such that nodes $A$ and $B$ are symmetric to each other, we can conclude that binary power allocation is optimal for node $B$ as well.

\section{Binary Power Optimality of Half-Duplex Node}

Next, we consider the binary power optimality with respect to the half-duplex node $C$. Since $P_{A}$ and $P_{B}$ are binary optimum, we can analyse the network under three scenarios as,

1) $P_{A}=P_{B}=0$

2) $P_{A}=P_{\max }$ and $P_{B}=0$ (or $P_{A}=0$ and $P_{B}=P_{\max }$ )

3) $P_{A}=P_{B}=P_{\max }$

In the first scenario, the optimum $P_{C}$ value is $P_{\max }$ as it will be the only transmitting node.

For the second scenario, let us consider the case where $P_{A}=P_{\max }$ and $P_{B}=0$ and re-express the sum rate optimization problem as,

$$
\begin{array}{ll}
\max _{P_{C}} \hat{R} & \\
\text { Subject to } 0 \leq P_{C} \leq P_{\max }, \\
\\
P_{A}=P_{\max }, \\
P_{B}=0 .
\end{array}
$$

Taking the partial derivative of (10) with respect to $P_{C}$,

$$
\frac{\partial \hat{R}}{\partial P_{C}}=\frac{a ́ P_{C}^{2}+2 \hat{b} P_{C}+\dot{c}}{\dot{d}},
$$


where,

$\dot{a}=G_{C D} G_{C B}^{2}$,

$\hat{b}=G_{C D} G_{C B} \sigma_{B}^{2}$,

$\dot{c}=G_{C D} \sigma_{B}^{4}+P_{\max } G_{A B}\left[G_{C D} \sigma_{B}^{2}-G_{C B}\left(\sigma^{2}+P_{\max } G_{A D}\right)\right]$, $\dot{d}=\left(\sigma_{B}^{2}+P_{C} G_{C B}\right)^{2}\left(\sigma^{2}+P_{\max } G_{A D}\right)$.

Similar to section III-C, we can clearly see that the values of $\dot{a}, \hat{b}$ and $\dot{d}$ are all positive and the sign of $\dot{c}$ is in-deterministic. However, analysing the second derivative of $\hat{R}$ at $\partial \hat{R} / \partial P_{C}=$ 0 we find

$$
\frac{\partial^{2} \hat{R}}{\partial^{2} P_{C}}{ }_{\mid \frac{\partial \hat{R}}{\partial P_{C}}=0}=\frac{2 a ́ P_{A}+2 \hat{b}}{\hat{d}},
$$

which is strictly positive. Therefore, similar to section III-C we can conclude that binary power allocation is optimum for $P_{C}$ under the second scenario.

In the third scenario, the partial derivative of (8) with respect to $P_{C}$ can be derived as,

$$
\frac{\partial \hat{R}}{\partial P_{C}}=\frac{\ddot{a} P_{C}^{4}+2 \ddot{b} P_{C}^{3}+\ddot{c} P_{C}^{2}+2 \ddot{d} P_{C}+\ddot{e}}{\ddot{f}},
$$

where,

$$
\begin{aligned}
\ddot{a}= & G_{C D} G_{C A}^{2} G_{C B}^{2}, \\
\ddot{b}= & G_{C D} G_{C A} G_{C B}\left(G_{C A} \sigma_{B}^{2}+G_{C B} \sigma_{A}^{2}\right), \\
\ddot{c}= & P_{\max } G_{C D}\left(G_{A B} G_{C A}^{2} \sigma_{B}^{2}+G_{B A} G_{C B}^{2} \sigma_{A}^{2}\right)+ \\
& G_{C D}\left(G_{C B}^{2} \sigma_{A}^{4}+4 G_{C A} G_{C B} \sigma_{A}^{2} \sigma_{B}^{2}+G_{C A}^{2} \sigma_{B}^{4}\right)- \\
& P_{\max }^{2} G_{A B} G_{B A} G_{C D} G_{C A} G_{C B}-P_{\max } G_{C A} G_{C B} \\
& \left(\sigma^{2}+P_{\max }\left(G_{A D}+G_{B D}\right)\right)\left(G_{A B} G_{C A}+G_{B A} G_{C B}\right), \\
\ddot{d}= & G_{C D} \sigma_{A}^{2} \sigma_{B}^{2}\left(G_{C B} \sigma_{A}^{2}+G_{C A} \sigma_{B}^{2}\right)+P_{\max } G_{C D} \sigma_{A}^{2} \sigma_{B}^{2} \\
& \left(G_{A B} G_{C A}+G_{B A} G_{C B}\right)-P_{\max } G_{C A} G_{C B}\left[\sigma^{2}+P_{\max }\right. \\
& \left.\left(G_{A D}+G_{B D}\right)\right]\left(G_{A B} \sigma_{A}^{2}+G_{B A} \sigma_{B}^{2}+P_{\max } G_{A B} G_{B A}\right), \\
\ddot{e}= & G_{C D} \sigma_{A}^{4} \sigma_{B}^{4}+P_{\max } G_{C D} \sigma_{A}^{2} \sigma_{B}^{2}\left(G_{A B} \sigma_{A}^{2}+G_{B A} \sigma_{B}^{2}+\right. \\
& \left.P_{\max } G_{A B} G_{B A}\right)-P_{\max }\left[\sigma^{2}+P_{\max } G_{A D}+P_{\max } G_{B D}\right] \\
& {\left[G_{B A} G_{C A} \sigma_{B}^{4}+G_{A B} G_{C B} \sigma_{A}^{4}+P_{\max } G_{A B} G_{B A}\right.} \\
& \left.\left(G_{C A} \sigma_{B}^{2}+G_{C B} \sigma_{A}^{2}\right)\right], \\
\ddot{f}= & \left(\sigma_{B}^{2}+P_{C} G_{C B}\right)^{2}\left(\sigma_{A}^{2}+P_{C} G_{C A}\right)^{2} \\
& \left(\sigma^{2}+P_{\max } G_{A D}+P_{\max } G_{B D}\right) .
\end{aligned}
$$

As we end up with a quartic equation without any visible factorization, providing a condition for real and non-negative $P_{C}$ such that $\partial \hat{R} / \partial P_{C}=0$ becomes too complex. In addition, as there can be more than one critical point such that $\partial \hat{R} / \partial P_{C}=0$, proving the convexity of $\hat{R}$ with respect to $P_{C}$ becomes impossible. Thus, we cannot prove the convexity of $\hat{R}$ with respect to $P_{C}$ to satisfy all three scenarios given above.

In the following, we consider the binary power optimality of the half-duplex node under three special cases, 1) symmetric network, 2) approximation by the arithmetic mean-geometric mean inequality, and 3) the low-SINR regime.
1) Symmetric Network: Let us consider a symmetric network under the third scenario where $G_{C A}=G_{C B}=G_{A D}=$ $G_{B D}=\epsilon, G_{A B}=G_{A B}=G_{C D}=G, \sigma_{A}^{2}=\sigma_{B}^{2}=\sigma^{2}+\beta$ and $P_{A}=P_{B}=P_{\max }$ and analyse the binary power optimality of node $C$. Taking the first derivative of $\hat{R}$ with respect to $P_{C}$ we write

$$
\frac{\partial \hat{R}}{\partial P_{C}}=\frac{\left(\epsilon P_{C}+\sigma^{2}+\beta+G P_{\max }\right)\left(\breve{a} P_{C}^{2}+\breve{b} P_{C}+\breve{c}\right)}{\breve{d}}
$$

where,

$$
\begin{aligned}
& \breve{a}=G \epsilon^{2}>0, \\
& \breve{b}=2 G \epsilon\left(\sigma^{2}+\beta\right)-P_{\max } G^{2} \epsilon, \\
& \breve{c}=G\left(\sigma^{2}+\beta\right)^{2}+P_{\max } G\left[G\left(\sigma^{2}+\beta\right)-2 \epsilon\left(\sigma^{2}+2 P_{\max } \epsilon\right)\right], \\
& \breve{d}=\left(\sigma^{2}+2 P_{\max } \epsilon\right)\left(\sigma^{2}+\beta+P_{C} \epsilon\right)^{3}>0 .
\end{aligned}
$$

Since $\epsilon P_{C}+\sigma^{2}+\beta+G P_{\max }$ and $\breve{d}$ are always positive, we can see that real and non-negative $P_{C}$ can only occur such that $\partial \hat{R} / \partial P_{C}=0$ when $\breve{a} P_{C}^{2}+\breve{b} P_{C}+\breve{c}=0$. Analysing the second derivative of $\hat{R}$ with respect to $P_{C}$ at $\partial \hat{R} / \partial P_{C}=0$ we get

$$
\frac{\partial^{2} \hat{R}}{\partial^{2} P_{C}}{ }_{\mid \frac{\partial \hat{R}}{\partial P_{C}}=0}=\frac{\left(\epsilon P_{C}+\sigma^{2}+\beta+G P_{\max }\right)\left(2 \breve{a} P_{C}+\breve{b}\right)}{\breve{d}} .
$$

Since the sign of (11) is in-deterministic, we cannot guarantee the convexity or the binary optimality of $P_{C}$. However, we can define a sufficient condition under which binary power allocation is optimum for $P_{C}$ as,

$$
P_{\max } \leq \frac{2\left(\sigma^{2}+\beta\right)}{G} .
$$

The above condition guarantees that $\frac{\partial^{2} \hat{R}}{\partial^{2} P_{C}} \geq 0$ at $\frac{\partial \hat{R}}{\partial P_{C}}=$ $0, \forall P_{C}$. Thus, the range $0 \leq P_{c} \leq P_{\max }$ can only have a global minimizer. Therefore, within this range $\hat{R}$ is either increasing or decreasing or convex with respect to $P_{C}$ proving that optimum power allocation is binary for $P_{C}$ under the condition given in (12). We would like to highlight that the $P_{\max }$ value in the derived sufficient condition does not depend on the interference $\epsilon$. Therefore, we can design a network such that binary power allocation is optimum for all nodes by simply controlling the noise power, the self-interference and the desired gain of each link.

2) Arithmetic mean-geometric mean approximation: According to the arithmetic mean-geometric mean inequality given by [20], the following holds

$$
\sum_{n=1}^{N} \frac{x_{n}}{N} \geq\left(\prod_{n=1}^{N} x_{n}\right)^{1 / N}
$$

where $x_{1}, x_{2}, \ldots, x_{N}$ are non-negative real numbers. Applying the above inequality we can approximate $\sqrt[3]{\hat{R}}$ by $\Delta$ where

$$
\begin{gathered}
\Delta=1+\frac{P_{B} G_{A B}}{3\left(\sigma_{A}^{2}+P_{C} G_{C A}\right)}+\frac{P_{A} G_{A B}}{3\left(\sigma_{B}^{2}+P_{C} G_{C B}\right)} \\
+\frac{P_{C} G_{C D}}{3\left(\sigma^{2}+P_{A} G_{A D}+P_{B} G_{B D}\right)} .
\end{gathered}
$$


Taking the second derivative of $\Delta$ with respect to $P_{C}$ we can write

$$
\frac{\partial^{2} \Delta}{\partial^{2} P_{C}}=\frac{2 P_{B} G_{A B} G_{C A}^{2}}{3\left(\sigma_{A}^{2}+P_{C} G_{C A}\right)^{3}}+\frac{2 P_{A} G_{A B} G_{C B}^{2}}{3\left(\sigma_{B}^{2}+P_{C} G_{C B}\right)^{3}},
$$

which is positive. Thus, $\Delta$ is convex with respect to $P_{C}$. Therefore, binary power allocation for half-duplex node $C$ is optimum for approximated sum rate under the arithmetic mean-geometric mean inequality.

3) Low SINR regime: In the low-SINR regime, the approximation $\log _{2}(1+S I N R) \approx \frac{S I N R}{\ln 2}$ which is obtained by Taylor expansion holds [21]. Therefore, the sum rate $R$ in (1) can be approximated by $\Theta$ where

$$
\begin{gathered}
\Theta=\frac{P_{B} G_{A B}}{\ln 2\left(\sigma_{A}^{2}+P_{C} G_{C A}\right)}+\frac{P_{A} G_{A B}}{\ln 2\left(\sigma_{B}^{2}+P_{C} G_{C B}\right)} \\
+\frac{P_{C} G_{C D}}{\ln 2\left(\sigma^{2}+P_{A} G_{A D}+P_{B} G_{B D}\right)} .
\end{gathered}
$$

Taking the second derivative of $\Theta$ with respect to $P_{c}$ we can write

$$
\frac{\partial^{2} \Theta}{\partial^{2} P_{C}}=\frac{2 P_{B} G_{A B} G_{C A}^{2}}{\ln 2\left(\sigma_{A}^{2}+P_{C} G_{C A}\right)^{3}}+\frac{2 P_{A} G_{A B} G_{C B}^{2}}{\ln 2\left(\sigma_{B}^{2}+P_{C} G_{C B}\right)^{3}},
$$

which is positive. Since second derivative is non-negative, $\Theta$ is convex with respect to $P_{C}$. Therefore, in the low SINR regime, binary power allocation for half-duplex node $C$ is optimum for the approximated sum rate.

In the next section, we have illustrated that for both the full-duplex network under the third scenario and the special symmetric network that does not satisfy the sufficient condition in (12), we can find an optimum $P_{C}$ value which is not 0 or $P_{\max }$. Thus, proving that binary power allocation is not always optimum for $P_{C}$ in general or even in the symmetric network.

\section{Simulation And Numerical Results}

In this section, we first consider a general network set up where $P_{A}$ and $P_{B}$ values are set to $P_{\max }=3.5$ as in the third scenario in section III-D. We assume normalized desired channels gains of $G_{A B}=0.81, G_{B A}=0.79, G_{C D}=0.9$ and interference gains of $0.25,0.25,0.3,0.1$ and 0.005 for $G_{C A}, G_{C B}, G_{A D}, G_{B D}$ and $\beta$ respectively. Noise power $\left(\sigma^{2}\right)$ is assumed to be 1 . Fig. 3 plots the sum rate $R$ versus $P_{C}$, the transmit power of the half-duplex node $\mathrm{C}$ for the above example scenario. We can see that the sum rate graph has both a global minimizer and a global maximizer within the range $0 \leq P_{C} \leq P_{\max }$. Thus, the optimum power allocation of $P_{C}$ is different from $\left\{0, P_{\max }\right\}$.

Next, we have considered a simple network with symmetric desired and interference channel gains as in the special case we considered in section III-D1. We have assumed that the desired gains between nodes are 1, interference gains between nodes are 0.25 , self-interference powers of both full-duplex nodes are 0.01 and noise power is 1 . The maximum power of each node is set to $3.1 \mathrm{~W}$, such that $P_{\max }$ is outside the required range in the sufficient condition in (12) as

$$
P_{\max }<=\frac{2\left(\sigma^{2}+\beta\right)}{G}=2.02 \text {. }
$$

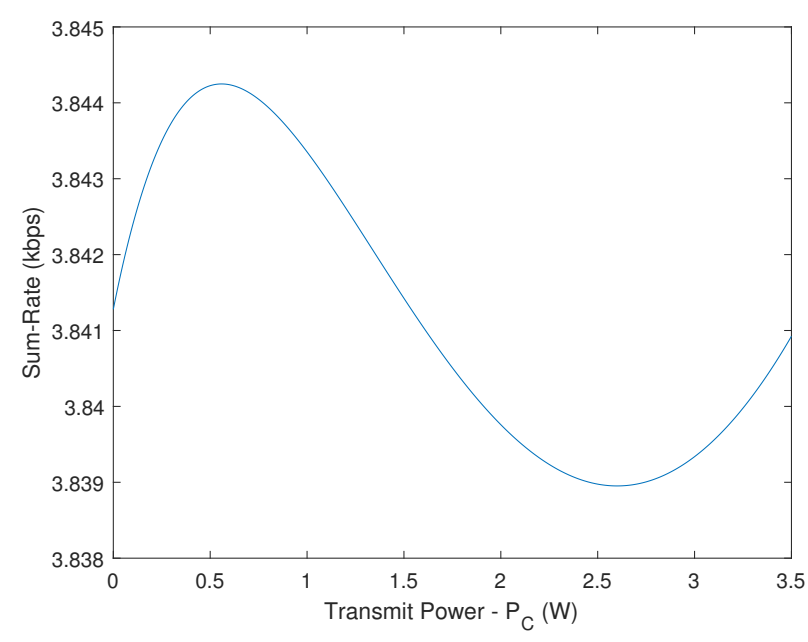

Figure 3: Sum rate vs. $P_{C}$ in general case

First, we consider binary power allocation and find the maximum sum rate as $4.053 \mathrm{kbps}$, which results when $P_{A}=$ $P_{B}=P_{C}=P_{\max }$. However, by solving this simple optimization problem, we find that the maximum sum rate is 4.05915 kbps which results when $P_{A}=P_{B}=P_{\max }$ and $P_{C}=0.94=0.3 P_{\max }$. Therefore, in this scenario, even though we have considered a simple symmetric network binary power allocation is not optimum for node $C$.

Finally, in Fig. 4 and 5, we consider a symmetric network where full-duplex nodes are transmitting with full power and plot the sum rate versus $P_{C}$. In Fig. $4, P_{\max }$ is selected within the sufficient condition in (12), while in Fig. 5, $P_{\max }$ is selected outside the sufficient condition in (12). We can clearly see that when $P_{\max }$ is within the sufficient condition, optimum power allocation is $P_{C}=P_{\max }$, thus binary power allocation is optimum. However, for the case where $P_{\max }$ is outside the sufficient condition, the graph is concave with a global maximum in between 0 and $P_{\max }$ which contradicts with binary power allocation.

\section{CONCLUSION}

We considered a simple network with four communicating nodes, two of them operating in half-duplex mode and the other two in full-duplex mode, and focused on maximizing the overall sum rate of the network with maximum power constraints on each node. We have shown that for the fullduplex nodes, binary power allocation remains optimal, but for the half-duplex node binary power allocation is not always optimal. However, for low SINR regime where the approximation $\log _{2}(1+S I N R) \approx \frac{S I N R}{\ln _{2}}$ holds and where the approximation by the arithmetic mean-geometric mean inequality is accurate, binary power allocation is optimum for the approximated sum rate. Furthermore, we have identified a requirement on the maximum transmit power that is allowed per node, such that the binary power allocation becomes optimum for the half-duplex node under the assumption of symmetric gains and interference. Obtained results can be further extended to 


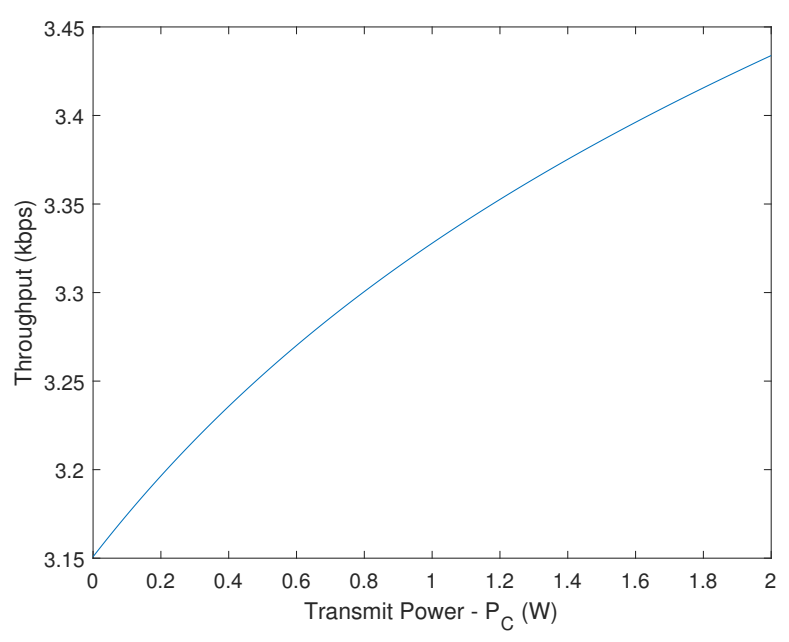

Figure 4: Sum rate vs. $P_{C}$ in symmetric network with $P_{\max }=2$

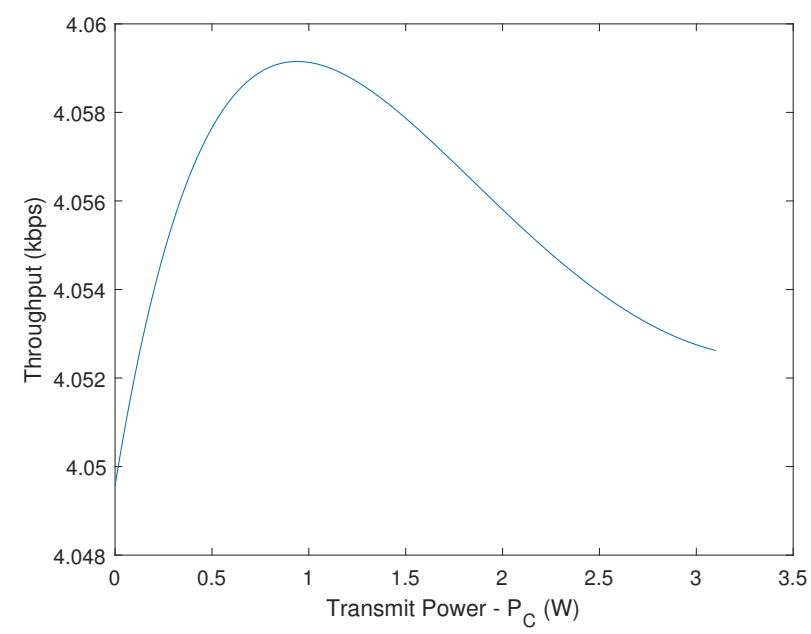

Figure 5: Sum rate vs. $P_{C}$ in symmetric network with

$$
P_{\max }=3.1
$$

a more general network setting through grouping of nodes using orthogonal frequency division multiplexing (OFDM) sub-carrier allocation. A future line of work would be to identify specific types of full-duplex networks where we can use binary power allocation to simplify the optimization of network sum rate with power control.

\section{REFERENCES}

[1] A. Goldsmith, Wireless Communications. Cambridge University Press New York, NY, USA, 2005.

[2] H. Inaltekin and S. V. Hanly, "Optimality of Binary Power Control for the Single Cell Uplink ," IEEE Transactions on Information Theory, vol. 58, no. 10, pp. 6484-6498, October 2012.

[3] M. Chiang, C. W. Tan, D. P. Palomar, D. O’Neill, and D. Julian, "Power Control By Geometric Programming ," IEEE Transactions on Wireless Communications, vol. 6, no. 7, pp. 2640 - 2651, July 2007.

[4] A. Gjendemsj, D. Gesbert, G. E. Oien, and S. G. Kiani, "Binary Power Control for Sum Rate Maximization over Multiple Interfering Links,"
IEEE Transactions on Wireless Communications, vol. 7, no. 8, pp. 3164 - 3173, August 2008.

[5] X. Qiu and K. Chawla, "On the performance of adaptive modulation in cellular systems ", IEEE Transactions on Communications, vol. 47, no. 6 , pp. $884-895$, June 1999.

[6] A. Babaei and B. Abolhassani, "A new iterative method for joint power and modulation adaptation in cellular systems," in Second IFIP International Conference on Wireless and Optical Communications Networks, 2005, Dubai, United Arab Emirates, June 2005.

[7] S. Jafar and A. Goldsmith, "Adaptive Multirate CDMA for Uplink Throughput Maximization," IEEE Transactions on Wireless Communications, vol. 2, no. 2, pp. 218 - 228, March 2003.

[8] N. Badruddin, J. Evans, and S. Hanly, "On Optimal Power Allocation for a Class of Interference Networks," in 2010 IEEE Global Telecommunications Conference GLOBECOM 2010, Miami, FL, USA, December 2010.

[9] S. R. Bhaskaran, S. V. Hanly, N. Badruddin, and J. S. Evans, "Maximizing the sum rate in symmetric networks of interfering links "' IEEE Transactions on Information Theory, vol. 56, no. 9, pp. 4471-4487, September 2010.

[10] R. Li, Y. Chen, and Y. Wu, "Binary Power Control for Full-Duplex Networks ", in 2016 IEEE 27th Annual International Symposium on Personal, Indoor, and Mobile Radio Communications (PIMRC), Valencia, Spain, September 2016.

[11] R. Zhang, M. Ma, D. Li, and B. Jiao, "Investigation on DL and UL Power Control in Full-Duplex Systems," in 2015 IEEE International Conference on Communications (ICC), London, UK, June 2015, pp. 1903-1907.

[12] J. Maraevi, J. Zhou, H. Krishnaswamy, Y. Zhong, and G. Zussman, "Resource Allocation and Rate Gains in Practical Full-Duplex Systems," IEEE/ACM Transactions on Networking, vol. 25, no. 1, pp. $292-305$, July 2017.

[13] C. Sexton, Q. Bodinier, A. Farhang, N. Marchetti, F. Bader, and L. A. DaSilva, "Coexistence of OFDM and FBMC for Underlay D2D Communication in 5G Networks," in 2016 IEEE Globecom Workshops (GC Wkshps), Washington, DC, USA, December 2016.

[14] L. J. Rodrguez, N. H. Tran, and T. Le-Ngoc, "Performance of FullDuplex AF Relaying in the Presence of Residual Self-Interference," IEEE Journal on Selected Areas in Communications, vol. 32, no. 9 , pp. 1752 - 1764, June 2014

[15] T. Riihonen, S. Werner, and R. Wichman, "Hybrid Full-Duplex/HalfDuplex Relaying with Transmit Power Adaptation," IEEE Trans. Wireless Commun., vol. 10, no. 9, pp. 3074-3085, 2011.

[16] T. M. Kim and A. Paulraj, "Outage probability of amplify-and-forward cooperation with full duplex relay," in 2012 IEEE Wireless Communications and Networking Conference (WCNC), Shanghai, China, April 2012.

[17] X. Hou, F. Liu, and Y. Liu, "A novel power allocation algorithm for D2D communications underlaying full-duplex cellular networks ," in 2017 3rd IEEE International Conference on Computer and Communications (ICCC), December 2017.

[18] D. Bharadia, E. McMilin, and S. Katti, "Full duplex radios," SIGCOMM Comput. Commun. Rev., vol. 43, no. 4, pp. 375-386, Aug. 2013. [Online]. Available: http://doi.acm.org/10.1145/2534169.2486033

[19] M. Jain, J. Choi, T. Kim, D. Bharadia, S. Seth, K. Srinivasan, P. Levis, S. Katti, and P. Sinha, "Practical, Real-Time, Full Duplex Wireless," in 17th annual international conference on Mobile computing and networking. ACM, 2011, pp. 301-312.

[20] P. Bullen, D. Mitrinovi, and P. Vasi, Means and their inequalities. Dordrecht : Springer, 1988.

[21] C. Edwards and D. E. Penney, Calculus and analytic geometry. Upeer Saddle River, NJ: Prentice-Hall, 1998. 


\section{University Library}

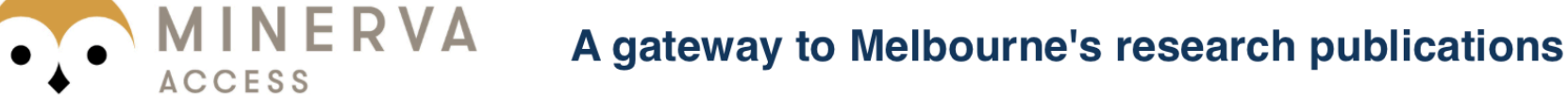

Minerva Access is the Institutional Repository of The University of Melbourne

Author/s:

Dayarathna, S;Senanayake, R;Evans, J

Title:

Binary Power Optimality for Two Link Full-Duplex Network

Date:

2020-05

Citation:

Dayarathna, S., Senanayake, R. \& Evans, J. (2020). Binary Power Optimality for Two Link Full-Duplex Network. Proceedings of the 2020 IEEE Wireless Communications and Networking Conference (WCNC), 2020-May, IEEE. https://doi.org/10.1109/ wcnc45663.2020.9120530.

Persistent Link:

http://hdl.handle.net/11343/241460 